\title{
La Comunicación organizacional interna en la organización educativa
}

\author{
Dra. Katia Papic Domínguez ${ }^{1}$
}

\section{RESUMEN}

En el presente trabajo se reflexiona sobre la función de la comunicación organizacional interna, dado que favorece el mantenimiento y el desarrollo de la organización educativa en el marco del desenvolvimiento de una gestión participativa. En la actualidad se la concibe como estrategia global, que posibilita atender las características y desafíos del contexto social y cultural y del sistema educativo de Chile, en el proceso de implementar la educación con calidad, equidad y participación.

Palabras clave: Comunicación organizacional, comunicación, organización educativa.

\section{Internal organizational communication in the educational organization}

\section{ABSTRACT}

This paper pretends to meditate about the internal organizational communication because it keeps up and develops the educational organization in the frame of a participative management. In this regard, should be noted that actually it is conceived as a global strategy that enables the characteristics and challenges of the social and cultural context and for the Chilean Educational System, in the process of implementing education with quality, equality, and participation.

Keywords: Organizational communication, communication, educational organization.

I Doctora en Comunicación organizacional. Académica Instituto Interdisciplinario en Pedagogía y Educación de la Universidad Católica Silva Henríquez. E-mail: kpapic@uchs.cl 


\section{Introducción}

Las principales condiciones de contexto mundial, que se consideran porque están potenciando la realización de cambios sustantivos en los modos de gestionar las organizaciones educativas, aluden a la relevancia de aplicar la comunicación organizacional interna en la promoción de la gestión escolar en los establecimientos educativos, para posibilitar el desarrollo y mantenimiento de las instituciones educativas en el siglo XXI en Chile, en el marco del fortalecimiento de la educación pública, de la mejora continua y de la educación con calidad, equidad y participación. Para reflexionar acerca de esta realidad se abordan, de manera general, los principales fenómenos de orden mundial, antecedentes relevantes del sistema educacional, de la comunicación organizacional interna, de la organización educativa y de la gestión escolar.

\section{Desarrollo}

\section{El contexto sociocultural: ciertos elementos claves}

Desde el enfoque social, Aguaded (2010, p. I) plantea hablar con cierta propiedad de una "era postmodernista", configurada por un conjunto de rasgos más o menos determinados que sirven para singularizar la sociedad de fines del milenio; asimismo, entre sus demarcaciones más características y propias, se identifican las etiquetas de "sociedad del consumo y del bienestar" y "sociedad de la información", dado que la precisan y constituyen de mejor manera.

Además, la globalización promueve la ruptura de las fronteras visibles entre las regiones y los Estados, y, a partir de este movimiento, se conforma la denominada "aldea global", referida principalmente a la mundialización de la economía y del comercio, y a la creación de un pensamiento único. También es preciso mencionar-acerca de la aldea global y la red económica- el empleo del idioma inglés y el uso de las tecnologías para favorecer la comunicación.

La convergencia de dichos procesos, al parecer, ha potenciado la generación de la cultura de la globalización, que se funda en actitudes 
con connotación identitaria como la de pertenecer y participar en una entidad social, y concretar un desarrollo a escala planetaria, que puede generar implicancias de tipo económico, político, social, educacional, entre otros, a nivel regional y local; además, desde este enfoque es posible considerar la "cultura de la globalización", porque genera un sistema de relaciones tendiente a conectar en red a la sociedad mediante el empleo de las tecnologías de la información y de la comunicación.

En relación con esta materia, desde el enfoque etnológico, Augé (2007, p. I) plantea cómo deben ser las relaciones en una cultura: en primer lugar, concebibles en términos de simbolizar un nexo entre las personas que se reconocen como integrantes de una colectividad y, en segundo, gestionables en el sentido de instituirse en organizaciones que las actualizan; incluso hoy está presente en la estructura y cultura del centro educativo la existencia de sistemas de relaciones orientadas a actualizar principios, valores y modos de actuar correspondientes a la cultura de la globalización.

Menciono la sociedad de la información porque alude a la transmisión de esta mediante el empleo de tecnologías de la información y de la comunicación, como la telefonía, la web y la televisión satelital. Respecto de ello, valoro, en consonancia con Delors (I997, p. 37), la forma en que las nuevas tecnologías han promovido la entrada de la humanidad en la época de la comunicación universal, debido a que, al eliminar la distancia, aportan de forma poderosa para crear las sociedades del mañana y no corresponderán a arquetipos del pasado. Abundando sobre este planteamiento, Mattelart (2003, p. I0) afirma que, frente a la definición particular y unívoca de "conocimiento", se opta por la expresión "sociedad de los saberes" para todas las personas y por todas las personas, con el objeto de nombrar el plan de sociedad equitativa, obteniendo así un beneficio de las nuevas tecnologías de la información y de la comunicación.

En particular, aprecio la sociedad de la información, siguiendo a Aguaded (2002, p. I3) cuando cita a Mattelart (I995, pp. I9 y ss.), porque conforma una "sociedad del flujo", en la cual la comunicación se transforma en un modo de ordenamiento del mundo, porque integra los medios y la circulación e intercambio entre los seres 
humanos; asimismo, Mattelart (I995, p. I09) alude al "culto a la red" y a la "ciudad comunitaria" con el objeto de abordar unas sociedades que incrementan el establecimiento de conexiones entre sí, mediante el empleo de las redes de la información y de la comunicación, cuya racionalidad trata el funcionamiento de modo mundial.

También Gámez (2007, p. I I) releva el gran desarrollo de las tecnologías y de la profesionalización de las prácticas, porque tornan a la comunicación un emblema de las sociedades del tercer milenio. Acerca de esto, sugiere que la comunicación se sustenta, de forma importante, en las nuevas tecnologías y en la profesionalización de las prácticas, en el sentido de posibilitar transmitir información de diversos tipos y a cualquier persona desde cualquier lugar y tiempo, con el objeto de satisfacer las necesidades de los usuarios, con relación a sus intereses y expectativas en el contexto de su desenvolvimiento personal, social y laboral. Asimismo, en el caso de las organizaciones educativas, señala que esta situación favorece su funcionamiento cotidiano y proyecta su visión de futuro mediante identificar, comprender y asumir las tendencias de las ciencias, de las tecnologías de la comunicación e información, de la economía, entre otras, mundial y regionalmente.

En este entorno social y cultural, el sentido más humano de la educación, según Hopenhayn (2006, p. 19), reside en su propiedad de imperativo para educar personas con mayor armonía y mejor disposición a la comunicación, y conformar un dinamizador de los aprendizajes para el trabajo y las nuevas maneras de aplicar saberes al campo productivo. También, señala la necesidad de potenciar en la escuela la comunicación entre hombres y mujeres, entre jóvenes de etnias o grupos socioculturales distintos, como aprendizaje cotidiano para la sociedad multicultural, de aceptación a la diversidad y coexistencia en la diferencia, dados los modos de relación entre estudiantes, y entre maestros y estudiantes, como asimismo la facultad de los profesores para fortalecer el desenvolvimiento humano y espiritual de sus alumnos. Este planteamiento configura una de las claves para que las nuevas generaciones puedan desarrollarse en una sociedad de progresiva complejidad y con requerimiento de seres humanos integrales. 
Hopenhayn señala que los cambios próximos formulan importantes desafíos al estilo y al contenido de la educación; pues nuevos modos culturales -como símbolos, fantasmas, deseos, proyectos vitalessurgen en la sociedad del riesgo, la sociedad mediática, la sociedad de gestión y la sociedad democrática; también, trazan retos al sistema educativo y a la preparación de las recientes generaciones, y todos estos componentes demandan un nuevo ánimo para el sentido más hondo de la educación: el desarrollo integral de los seres humanos.

\section{Sistema escolar: principales componentes del contexto}

Valoro la organización escolar, en cuanto componente del sistema educativo nacional, porque es objeto de los lineamientos de la Reforma Educacional, ya que desde 20I4, parafraseando al Ministerio de Educación (MINEDUC) (2014, p. 2), se establece como ejes del sistema escolar, entre otros, el de educación pública de calidad y el de profesión docente moderna. En torno a la educación pública de calidad, se observa el planteamiento de MINEDUC sobre la nueva institucionalidad en la educación parvularia, para incrementar su cobertura a nivel nacional; también, que la nueva educación pública integra la creación de nuevos servicios locales de educación, dirigidos a administrar las escuelas municipales con una alta participación de las comunidades, y otorga apoyo técnico, administrativo y financiero a los establecimientos educacionales. Asimismo, expresa que el itinerario de apoyo y fortalecimiento a la educación pública 2014-2018 enfoca la realización de mejoras específicas en las escuelas y liceos públicos, en los campos de infraestructura, conectividad digital, talleres, equipos deportivos y artísticos, cambio pedagógico, desenvolvimiento de potencialidades docentes y directivas, y asistencia a la participación y formación estudiantil.

Por tanto, en la actualidad existe en Chile la necesidad de promover el desarrollo de la gestión de los establecimientos educativos para mejorar los aprendizajes. Sobre este tema, el MINEDUC ha formulado como uno de los grandes desafíos modificar los estilos de gestión en los establecimientos educacionales, con el objeto de 
mejorar la calidad de los procesos educacionales. Considero en gran medida como origen de los problemas de aprendizaje los procesos educativos que se llevan a cabo en las escuelas; en relación con esto identifico como algunos de los factores que inciden en la situación de baja calidad de los resultados educativos las prácticas pedagógicas frontales, la desarticulación curricular, los tipos de prácticas y las falencias en la gestión.

Siguiendo esta línea de trabajo, MINEDUC (2015, p. I) plantea que la Reforma Educacional de 2015 destaca el área de convivencia escolar y participación, pues se pretende vigorizar el clima escolar y perfeccionar los ambientes en las escuelas y liceos públicos. Además, singulariza, en el ámbito de asistencia a los docentes de la educación pública, programas orientados a entregar apoyo a los docentes, a la gestión de directores y a robustecer el liderazgo.

Por otra parte, respecto de abordar el desafío de potenciar los establecimientos educativos municipales, refieren UNESCO y UNICEF (2014, pp. 93-99) que Raczynski y Salinas (2008) relevan la toma de decisiones de tipo pedagógico, en particular para ubicarla en el espacio local, con el fin de asegurar el enlace entre la política general y los establecimientos educativos con un nivel más alto de compromiso de profesores y directivos en la indagación de mejoras.

Finalmente, tengo presente, siguiendo a UNESCO y UNICEF (2014, pp. I02-I03) al referenciar a Eyzaguirre y Fontaine (2008, p. I7), promover el estudio de las variables propias de las escuelas para entender las desemejanzas de rendimiento, con independencia del nivel socioeconómico de los estudiantes, arguyendo que estos influencian los resultados de aprendizaje en la misma medida que los factores sociofamiliares, y reconocer la posibilidad de tratarlo empleando políticas de mediano plazo, como los "modelos de buenas prácticas”.

Asimismo, plantean estos autores que, en el examen de los factores endógenos del sistema, cabe consignar al tema docente; además, citan a Vegas (2008) para distinguir las variables que inciden en el desempeño profesional de los profesores, tales como disponer de infraestructura y materiales apropiados; comprender los resultados 
que se espera de ellos; conocer las normas de selección y pautas de asignación; disponer de información acerca de expectativas y progresos; aplicar una labor continua de elaboración de estrategias de aprendizaje; contar con la facultad para usar su juicio en la definición de estrategias y políticas de incentivos dirigidas a mantener los buenos docentes.

También, de acuerdo a la cita de Eyzaguirre y Fontaine, el análisis de la institución escolar permite pormenorizar disimilitudes en los resultados de aprendizaje, pues estos están vinculados a las diferencias de gestión, dado que los establecimientos educativos de mayores rendimientos manifiestan equipos constituidos por el sostenedor y los directivos, focalizados en promover una labor centrada, sistemática y coordinada, orientada a implementar prácticas de gestión y actividades pedagógicas.

Con relación a esta materia, es necesario tener en cuenta los Estándares Indicativos de Desempeño para los Establecimientos Educacionales y sus Sostenedores, siguiendo a MINEDUC (20I4, p. 5), porque posibilitan considerar la evaluación de los procesos de gestión escolar de las organizaciones escolares y sus sostenedores, cuya finalidad es asistir y orientar a los establecimientos educacionales en la puesta en práctica de sus procesos de mejora continua, contribuyendo de esta forma al logro del objetivo de garantizar una educación de calidad para la totalidad de los niños y jóvenes de Chile.

Para ampliar esta perspectiva, subyacen en todo proceso de evaluación de la organización educativa, en el marco de la mejora continua, las ideas de comunicación organizacional interna y de gestión de la organización escolar, por cuanto es conveniente considerar la concepción e institucionalización de un cambio, innovación y/o mejora mediante instancias de estudio en y para la práctica, que conlleven el establecimiento de consensos y de aprendizajes en los profesores directamente involucrados en la situación en cuestión y del centro educativo en general.

En este contexto, la Ley General de Educación, publicada en el Diario Oficial el I2 de septiembre de 2009, artículo $N^{\circ}$ 6, de acuerdo con MINEDUC (2009, p. I), señala como deber del 
Estado garantizar una educación de calidad y procurar que sea impartida a todos; por esto corresponde al Ministerio de Educación, al Consejo Nacional de Educación, a la Agencia de Calidad de la Educación y a la Superintendencia de Educación, en el contexto de sus competencias, la administración del Sistema Nacional de Aseguramiento de la Calidad de la Educación. En tal sentido, la mejora focaliza el tratamiento de la calidad y equidad educativa, los procesos y resultados de los aprendizajes de los estudiantes y la gestión institucional participativa.

La calidad implica la aplicación del criterio de exigencia y de apoyo brindado a la escuela para establecer el cumplimiento de los aprendizajes esperados, correspondientes a cada semestre y nivel según el currículo nacional. Sobre esta materia, según MINEDUC (2009, p. 2), en la Ley General de Educación, publicada en el Diario Oficial el 12 de septiembre de 2009, la calidad de la educación trata de confirmar que la totalidad de los estudiantes, con independencia de sus condiciones y contextos, logren concretar los objetivos generales y los estándares de aprendizaje que se determinen del modo que define la ley.

En torno a la calidad de la educación, en el desarrollo de las funciones del Sistema Escolar Nacional y por ende del establecimiento educativo, señala MINEDUC (2009, p. I) que en la Ley General de Educación de 2009, publicada en el Diario Oficial el 12 de septiembre, artículo $N^{\circ} 2$, se postula atender principalmente el derecho a la educación, descrito como un proceso de aprendizaje permanente, que contempla las diversas etapas de la vida de las personas y cuyo propósito es alcanzar el desarrollo espiritual, ético, moral, afectivo, intelectual, artístico y físico, mediante la transmisión y el cultivo de valores, conocimientos y destrezas.

Asimismo, apunta MINEDUC, de acuerdo con la Ley General de Educación, publicada en el Diario Oficial el I2 de septiembre de 2009, artículo $N^{\circ} 3$, que el sistema educativo chileno se conforma sobre la base de los derechos garantizados en la Constitución, los tratados internacionales vigentes ratificados en Chile y, en particular, en el derecho a la educación y en la libertad de enseñanza, lo que conlleva el deber de financiar un sistema gratuito orientado a asegurar el acceso de la población a las enseñanzas básica y media y a la educación parvularia. 
Por otra parte, en la Ley General de Educación, siempre según MINEDUC (2009, p. 2), la equidad se define como el sistema enfocado a garantizar a todos los alumnos la disponibilidad de las mismas posibilidades de recepcionar una educación de calidad, con particular atención de las personas o grupos con mayores necesidades de especial apoyo.

La Constitución política, indica UNESCO (2010/I I, p. 4), cuyo texto sistematizado es promulgado mediante Decreto $\mathrm{N}^{\circ} \mathrm{IOO}$ publicado en el Diario Oficial el 17 de septiembre de 2005, expresa en el art. I9, $\mathrm{N}^{\text {os }}$. I0-II el derecho a la educación y libertad de enseñanza. Por otra parte, la Constitución modificada en 2003, a través de la Ley $N^{\circ}$ 19.876, publicada en el Diario Oficial el 22 de mayo de 2003 de reforma constitucional, garantiza 12 años de escolaridad obligatoria y gratuita para todos los jóvenes del país hasta los 2I años; también considera a "kínder" como obligatorio, pero no como necesario para ingresar a primero básico.

En el ámbito de la participación, el artículo I4 del D.F.L. $\mathrm{N}^{\circ}$ I del Estatuto Docente, de acuerdo a MINEDUC (1997, p. I) indica que los profesionales de la educación tendrán derecho a participar, con facultad consultiva, en el diagnóstico, planeamiento, ejecución y evaluación de las actividades del establecimiento educativo correspondiente, y de sus vinculaciones con la comunidad; asimismo, tendrán derecho a ser consultados en los procesos de proposición de políticas educacionales en los diferentes niveles del sistema escolar.

En este contexto, parafraseando a Escudero (2006, p. I2), la participación puede estimarse como un valor transversal a los sistemas educativos públicos, en cuyos diversos niveles de poder y de decisión debe estar presente, y en toda la red de actores involucrados, disponiendo cada uno sus respectivos ámbitos de competencia; en tal sentido, la participación es necesaria para acordar entre diversos actores un enfoque de responsabilidades compartidas y concertadas, para asegurar el cumplimiento del derecho de todos a una buena educación (Escudero, 2006a).

Por otra parte, el derecho a la educación de calidad se relaciona con el derecho a la libertad de opinión y de expresión, por cuanto 
la Asamblea General de las Naciones Unidas (2009, p. I), en la Declaración de los Derechos del Hombre, artículo I9, explicita que todo persona posee el derecho a la libertad de opinión y de expresión; este integra, principalmente, indagar y recepcionar informaciones $y$ pareceres junto con la capacidad de difundirlos, sin restricción de límites y empleando algún medio de expresión.

En este marco de derechos humanos, es atingente, según Álvarez (2007, p. I), pormenorizar que la comunicación interna permite cumplimentar el derecho a la información y a la libertad de expresión de los empleados. En síntesis, la comunicación interna busca el éxito organizacional, impulsando el talento y propiciando el conocimiento al interior de una organización. La naturaleza de la comunicación horizontal, apunta Beltrán (20II, p. 30), garantiza que todas las personas tienen el derecho a comunicarse con el propósito de satisfacer sus requerimientos de comunicación, mediante el goce de los recursos de la comunicación.

En tal sentido, la Asamblea General de las Naciones Unidas (2009), en la Declaración de los Derechos del Hombre, artículo 26, expresa que todo individuo tiene derecho a la educación, con gratuidad y obligatoriedad de la instrucción elemental y fundamental. También, que la educación tendrá como finalidad el desarrollo pleno de la personalidad del hombre y el fortalecimiento del respeto a los derechos humanos y a las libertades fundamentales; promoverá el entendimiento, la tolerancia y la amistad entre todos los países y todos los conglomerados étnicos o religiosos.

En este contexto, la participación en la organización educativa cobra relevancia desde lo planteado en la Reforma Educacional, en la Ley General de Educación y en la Constitución, respecto principalmente de la educación con calidad y equidad. Por lo tanto, debe promoverse un proceso de mejora educativa integral en el que la comunidad educativa, compuesta por directivos, docentes, asistentes de aula, estudiantes, sostenedores, apoderados y familia, esté convocada a ser protagonista. En este sentido, la participación se refiere al reconocimiento de la vinculación de diversos actores en la tarea educativa y, de tal manera, en el mejoramiento de la calidad de la enseñanza y de los aprendizajes esperados de los estudiantes. 
Respecto de esta referencia, tanto la gestión descentralizada como la política educativa que alude a la participación constituyen su marco y su horizonte, pues se orientan a flexibilizar el tipo de relaciones que la escuela establece y mantiene con el sistema y su entorno, tanto interno como externo, y pretenden promover la transferencia de responsabilidades, destinadas a elaborar estrategias dirigidas a dar respuesta a problemas fundamentales de la sociedad y a mejorar la eficacia y eficiencia, mediante la puesta en práctica de acciones pertinentes y relevantes; así, la descentralización refleja el interés de propiciar cambios y/o acciones de mejora desde las propias organizaciones escolares.

Por último, el sistema educativo chileno, según el Ministerio de Educación (2009, p. I), de acuerdo con la Ley General de Educación $N^{\circ}$ 20.370, publicada en el Diario Oficial el I2 de septiembre de 2009 , artículo $3^{\circ}$, se origina en los siguientes principios: b) Calidad de la educación, por cuanto esta debe garantizar que todos los alumnos y alumnas, con independencia de sus condiciones y circunstancias, logren alcanzar los objetivos generales y los estándares de aprendizaje que se determinen en la ley; c) Equidad del sistema educativo, en tanto el sistema debe tender a asegurar que todos los estudiantes posean las mismas oportunidades de recibir una educación de calidad; g) Participación, porque los integrantes de la comunidad educativa gozan del derecho a ser informados y a participar en el proceso educativo de acuerdo con la normativa vigente. Además, en los principios de universalidad y educación permanente; autonomía; diversidad; responsabilidad; flexibilidad; transparencia, integración; sustentabilidad e interculturalidad.

Por tanto, estimo imperativo, a partir de los requerimientos y desafíos formulados en la Reforma Educativa, en la Ley General de Educación y en la Constitución política, pensar en la participación coordinada de los integrantes de la comunidad educativa como una estrategia global de la organización educativa, dirigida a la consecución de los fines educativos, en condiciones de calidad y equidad, en el marco del derecho a la educación y a la libertad de opinión y de expresión.

Con relación a este planteamiento, en el siglo XXI y en pleno surgimiento de la sociedad de la información y de la comunicación, 
Bataller (2006, pp. 27-28) indica que no se puede ni debe soslayar la relevancia de la participación como estrategia de perfeccionamiento continua de la calidad educativa, porque en la medida en que se potencie la participación de todos los agentes educadores es posible adelantar hacia la concreción de metas y objetivos cada vez más exigentes, justos y solidarios.

En este contexto de requerimientos y desafíos de carácter sociocultural y educativo, es necesario tener en cuenta, en consonancia con Fernández, Álvarez y Herrero (2002, p. 76), que en la organización escolar es inexistente la comunicación de la organización, porque se transforma en órdenes a cumplir, sin el necesario conocimiento de los propósitos de las mismas.

\section{La comunicación organizacional interna: función de la organización educativa}

La educación debe brindar acceso a los medios de información y de producción, siguiendo a López, Sánchez, Murillo, Lavié y Altopiedi (2003, p. 37); por ello, este paso se está transformando en un componente clave que dota de oportunidades o agrava situaciones de exclusión; incluso tiene en cuenta que la educación, como sistema de comunicación abierto, incide y se deja influir por el entorno social y tecnológico en el que está localizado.

Respecto de la importancia creciente de la comunicación en la organización educativa y acerca de la aplicación de la comunicación organizacional interna en entidades educativas, Papic (2016) detecta las líneas de investigación de la comunicación como factor del liderazgo en el establecimiento educativo, además de las implicancias del uso de las tecnologías de la comunicación e información en el centro educativo, la comunicación como variable de la cultura, la comunicación como principio de gestión de las universidades y la comunicación organizacional.

De modo sustantivo, la comunicación interna comprende, siguiendo a Villafañe (2002, p. 238), una función de orden estratégico. La comunicación interna, parafraseando a Zaragoza (2009, p. 87), es una realidad que implica la participación de los empleados en un 
proyecto organizacional compartido; la divulgación de información mediante canales rápidos y eficaces; la recolección de información utilizando instrumentos para asegurar una doble dirección de información. Además, ocupa los primeros lugares en las agendas de los responsables de comunicación y de los directivos de empresa.

Abundando sobre esta disciplina, en el marco de la información, la comunicación interna es la que se concreta de preferencia en el hecho comunicativo al interior de la organización educativa (Carnicero, p. 28).

En el campo de las organizaciones, la comunicación promueve la relación entre los integrantes de la organización con el sistema de objetivos definidos. En estos términos, Emanuelli (2000, p. I) sostiene, a través de Annie Bartoli, que la organización y la comunicación están vinculadas para el funcionamiento de la empresa y motivadas por los deseos de alcanzar resultados; asimismo, plantea que dicho vínculo tiene dos componentes: el primero es el político, conformado por los medios de una estrategia global en la que inciden, y el segundo es el sociocultural, compuesto por los valores, las representaciones y los fenómenos de poder que influencian la organización y la comunicación.

La comunicación interna, indica Weil (I992, pp. 209-2II), está integrada por dos dimensiones: una conforma la gestión de los sistemas de información, visualizada como una función operativa, cuantitativa y orientada a garantizar el tratamiento, la divulgación y circulación de la información en la organización; la otra, en cambio, constituye una función de incentivo, cualitativa y dirigida a alentar, completar o atender el quiebre de las redes precedentes. Por otra parte, la comunicación interna, al decir de este especialista, aporta a la gestión una garantía de mejor integración y sinergia de los trabajos, a través de irrigar y alimentarse de todos los servicios.

También en esta materia, la comunicación interna, siguiendo a Castillo (2004, p. 27I), es el tipo de comunicación orientado hacia los públicos internos de la organización. Rodríguez (I99I, p. 32) la define como agregado de acciones realizadas en una organización, con el objeto de alcanzar la generación y el mantenimiento de relaciones adecuadas con y entre sus integrantes, mediante el empleo de diversos 
medios de comunicación que los conserven informados, unidos y estimulados, para aportar con su labor a la consecución de los objetivos organizacionales. Por tanto, de acuerdo con este especialista, la comunicación interna constituye un canal de comunicación entre los integrantes de la entidad organizativa, porque mediante ella se produce un flujo de comunicación que circula en todos los sentidos para informar y disponer de información, y se lleva a cabo mediante canales formales y también relaciones informales.

Así, la comunicación en la institución, en palabras de Carnicero (2005, p. 19), es la totalidad de aquello destinado a disminuir la incertidumbre, posibilitando elegir y desempeñarse de modo que la entidad organizativa disponga de alternativas de mantenimiento y de crecimiento en consonancia con su planificación.

A partir del planteamiento precedente, la comunicación organizacional interna, en particular su orientación a los públicos internos, cobra significado en la organización educativa. Antúnez (2000, p. I5), siguiendo a García Hoz, la concibe, desde su significado institucional, como la comunidad constituida mayoritariamente por maestros y estudiantes y aplicada específicamente a la educación.

En la organización educativa tanto la estrategia como el proceso comunicativo están dirigidos a informar, motivar y conectar a todos los integrantes de la comunidad educativa. En tal sentido, la comunicación se configura, apuntan López et al. (2003, pp. 25-27), como un proceso que, luego de conformar el sistema social, continúa mientras se mantiene la interacción originaria entre al menos dos sujetos y, además, en su transitar, deja vestigios. Conciben al sistema social como preferentemente histórico, porque deja registro histórico que incide en el presente; estos signos pueden ser visualizados como malla o estructura, cuyo inicio se realiza durante su construcción y opera como sustento todo nuevo acto comunicativo. Así, las estructuras son, de manera simultánea, productoras y generadas por el proceso comunicativo; además, sugieren que la escuela selecciona su propia estrategia de acuerdo con su particular universo de significados y según su correspondiente red de relaciones o posiciones, desde las cuales se comunican los participantes de la misma. 
En el ámbito del desarrollo del dinamismo del sistema social de la organización educativa se debe acoger los objetivos de la comunicación organizacional interna en los procesos que sustentan su desenvolvimiento. Estos propósitos, de acuerdo con Álvarez (2007, p. I), corresponden a los de mejora de la imagen de la organización entre los integrantes de su personal, al entendimiento de la realidad organizacional, a la coherencia de la actividad laboral, a la promoción de la cultura de la entidad organizacional, a la moderación de la "rumorología", al tratamiento de situaciones de crisis, a la estimulación de la innovación, a la promoción de la calidad del proceso productivo y, en particular, al logro adecuado de los procesos de cambios internos. Desde estas finalidades, este autor reconoce que la comunicación interna permite cumplimentar el derecho a la información y a la libertad de expresión de los empleados. También, favorece el ejercicio del mismo derecho en los demás integrantes de la comunidad educativa en el espacio interno de la entidad organizativa.

Respecto de esto, desde el enfoque de la comunicación organizacional los participantes de la comunidad educativa constituyen públicos internos. Sin embargo, los públicos pueden corresponder a tres tipos, siguiendo a Costa (2010, p. I4) al parafrasear a Losada Díaz (2002): a los públicos internos, que incumben a la organización y, por tanto, mantienen una relación directa y permanente con la misma; a los públicos externos, que no pertenecen a la entidad organizativa y desenvuelven una relación esporádica, y a los públicos intermedios, que no pertenecen a la organización pero son afectados de manera directa por su gestión y toman parte de ella de modo relativamente activo.

Continuando con esta materia, para atender en la gestión de la organización escolar cabe definir al "público objetivo". Carnicero (2005, p. 29) lo visualiza como los destinatarios/receptores y los concibe como usuarios; de manera simultánea, consigna al público subjetivo o integrantes asimilados a la propia entidad organizativa o empleados.

El carácter de función estratégica de la comunicación organizacional interna queda de manifiesto cuando se establece la asociación entre los objetivos y los públicos internos. Respecto de esta, Villafañe (2002, p. 238), la comprende como asistir de forma estructural el 
proyecto de empresa, en el marco de la orientación estratégica de la organización, a partir del enfoque del management. Sin embargo, plantea que la importancia de la comunicación interna se incrementa en forma proporcional a la valoración que la empresa otorgue a los recursos humanos en su desenvolvimiento y competitividad.

\section{Organización educativa: comunicación organizacional interna base de la gestión participativa}

La comunicación, apunta Capriotti (2009, p. 43) a través de la cita de Tompkins (1984), configura las organizaciones, porque estas se comprenden como sistemas de personas en interacción y sumergidas en un proceso de reproducción y de recreación de la organización, por medio de la comunicación. Siguiendo esta línea de pensamiento, identifica a autores como Taylor (1993, 1995) y Stohl (1995) para definir la organización como un sistema de comunicación que se concreta mediante el discurso elaborado por un grupo de actores; $y$, al interior de una organización, la comunicación ya no irradia la realidad, sino que la produce y manifiesta el proceso de organización (Putnam et al., I996; Tompkins y Wanca-Thibault, 200I).

Además, ante esta situación, la comunicación, de acuerdo con Chen y Reigeluth (20I0, p. 252), configura una de las variables de mayor trascendencia que definen las contribuciones de los participantes en cualquier trabajo de equipo, en particular sobre una labor de alta complejidad como un proceso de transformación sistémica en todo el distrito escolar.

El establecimiento de este tipo de comunicación, siguiendo a Capriotti, se sustenta en dispositivos y actividades dirigidos a apoyar la libre circulación y el intercambio de información en el medio interno de la organización; apreciar en la organización que todos sus integrantes se encuentran implicados tanto en la comunicación como en el intercambio de información sobre el desarrollo de su actividad; visualizar el intercambio de información con un carácter multidireccional, en sentido ascendente, descendente y horizontal, por medio del diálogo enfocado en instalar un sistema simétrico de comunicación. Este enfoque de intercambio y de diálogo aborda 
como método básico la comunicación directa y el contacto personal entre los integrantes de una organización.

En este contexto, Gairín (1996, p. I98) identifica el sistema relacional como variable clave en la organización escolar. Además, señala que en el sistema relacional se encuentra la persona humana como el componente esencial de cualquier organización, porque actuar sobre la persona implica incidir en lo más importante y permitir, por tanto, los cambios más sustantivos y permanentes. Por último, expone que en el sistema relacional es trascendente el rol de los directivos y la relevancia de las estrategias de dinamización (Gairín, 1992), en particular su influencia en los procesos de comunicación, participación, toma de decisiones y atención de conflictos.

En una institución educativa de calidad, parafraseando a Gento y Montes (2010, pp. I8-19), en el abordaje metodológico educativo se tiene en cuenta la confluencia de principios elementales, como la consagración planificada, la adecuación a individuos y entorno, el fomento de potencialidades, la generación de un propicio clima interrelacional y la vinculación con otras entidades. Respecto del abordaje metodológico educativo, cabe mencionar lo detectado por Papic D. (2010), dado que se aprecia una tendencia compartida respecto de la adscripción de la referida función a los cargos directivos.

Para propiciar y establecer una estrategia global de participación coordinada de los integrantes de la comunidad educativa, se debe contemplar una formulación estratégica de comunicación interna que, de acuerdo con Capriotti (2008, p. 7I), debe contemplar una política de información enfocada a obtener datos, tanto para el trabajo como para conformar un contexto cultural de las representaciones y los valores compartidos, de manera de posibilitar la supresión de las representaciones antagónicas.

En la organización educativa, la gestión de una estrategia global de participación coordinada favorece el desenvolvimiento de los procesos tendientes a concretar los diversos objetivos consignados en la planificación estratégica. En tal sentido, cabe valorar el alcance de la comunicación organizacional interna y de su eficacia en el 
desarrollo del proyecto educativo institucional. La eficacia de la comunicación interna, indica Del Pozo (2004, p. 262), se encuentra asegurada en parte por el estilo de dirección y, en la estructura formal de la entidad organizativa, por un estilo de dirección participativo fundado en la confianza y en la comunicación, en el levantamiento de canales formales e informales dirigidos a combinar las diversas estructuras organizacionales, dado que esto beneficia la eficacia de la comunicación interna en la organización, porque "dirigir" significa integrar, representar, escuchar y construir equipo.

En particular, en los escenarios y procesos organizativos, citando a Carnicero (2005, p. I0), se manifiestan ciertos valores y preferencias por lo que se realiza y por los modos empleados para comunicarlo. Con relación a los modos, cabe observar lo detectado en la investigación de Papic (2010), dado que, de los directivos, una amplia mayoría, 84,6\%, emplea canales físicos de comunicación en calidad de soportes para transmitir información de manera directa a las personas; la gran mayoría, 92,3\%, afirmó usar canales personales de comunicación en la comunicación organizacional para posibilitar la información general de la organización. Por tanto, se distingue una tendencia a usar canales tanto físicos como personales en el desarrollo del flujo de información en el contexto personal y organizacional.

En este contexto, destaca en la aplicación de la comunicación interna, de acuerdo con Herranz (2007, p. II), enfocarse hacia resultados tales como la eficacia y calidad en la labor ejecutada, al compromiso, la conciliación y la motivación de los empleados por medio de la difusión de los valores de la entidad, para fortificar el sentido de unidad, la capacidad de innovación, con creatividad y participación y mediante canales propicios; a la flexibilidad y dinamismo interno con base en iniciativas y medidas encauzadas a fomentar las relaciones de colaboración y coordinación entre los equipos de trabajo y unidades; a fortalecer la cultura corporativa y a promover un clima laboral adecuado y participativo entre los públicos internos y la base social.

La comunicación interna conforma una herramienta de gestión eficaz para la entidad organizativa, según Ordeix (2009, p. 59), orientada a fortalecer su cultura organizativa mediante la aplicación 
de la habilidad de adecuación de los mensajes a su contexto cultural, a sus valores y a sus integrantes. Además, la comunicación interna en la organización manifiesta, según este autor, en calidad de requerimiento, desenvolver o considerar que la comunicación permite impedir reiteraciones en los procesos de gestión; brindar consistencia y seguridad tanto a las actividades como a los procesos de cambio; ordenar los objetivos individuales con los colectivos; generar una cultura institucional consolidada; incidir en la concreción de los objetivos estratégicos de la organización; unir eficiencia de la comunicación con la contribución del personal a la entidad, y favorecer en los trabajadores la aceptación de la relevancia y del requerimiento de dirigir los procesos comunicativos, en particular los enfocados a agregar valor a la entidad organizativa y a su marca.

A partir de lo señalado, valorar la comunicación organizacional interna como instrumento eficaz de la gestión del establecimiento educativo atañe a lo detectado en la investigación de Papic (2010), en la cual los directivos, en una amplia mayoría, 88,5\%, declara conformidad con usar la comunicación interna con el objeto de socializar la misión a los miembros de la comunidad educativa; la mayoría, 69,3\%, manifestó adhesión a la práctica de la comunicación interna para implicar a los públicos internos en el desenvolvimiento de la visión organizativa; un porcentaje levemente inferior al total, $96,2 \%$, manifestó conformidad con el uso de la comunicación interna con el objeto de que los actores de la comunidad educativa conozcan el motivo y la manera de realizar su trabajo.

Estos alcances de la comunicación organizacional interna cobran relevancia en su aplicación en el espacio de las instituciones escolares, porque, como afirma Carnicero (2005, p. 2I), enfocan la comunicación en el subsistema social y en su adaptación a los requerimientos de la sociedad, en el cambio e innovación desde la perspectiva del avance y en el sentido de identidad; inclusive, en el marco comunitario, enfatizan el establecimiento de relaciones con los integrantes de la comunidad y, en el contexto de organización y de gestión, posibilitan enfocar el desarrollo de la gestión y de la dirección. 
Carnicero también incluye, desde el prisma de la gestión y de la dirección, aportaciones con la articulación de los participantes en la aplicación del gobierno, con la ejecución de los procesos de toma de decisiones, con la resolución de conflictos y con la preparación del proyecto educativo institucional.

Respecto de la contribución de la comunicación organizacional interna a la administración de la gestión y de la dirección, se debe estimar la gestión escolar en cuanto constituye, según el Ministerio de Educación (2005, p. 27), la actuación profesional y técnica dirigida al establecimiento, de condiciones tendientes a favorecer el desenvolvimiento de buenas prácticas por parte de los actores, con relación a las metas institucionales y educacionales compartidas en la organización escolar.

Abundando sobre esta materia, corresponde mencionar la gestión educativa. UNESCO (2007, p. 6) la concibe como entender y valorar una nueva manera de comprender y de dirigir la organización escolar, porque estima como una de sus bases el cálculo estratégico situacional, el cual debe preceder, presidir y acompañar esa actividad de carácter educativa, para constituir el trabajo cotidiano de la enseñanza como en un proceso práctico creador de decisiones y de comunicaciones específicas. Respecto del proceso de comprender y de dirigir la organización educativa, en la investigación de Papic (20I0) se detecta una tendencia a incluir en el flujo de comunicación informaciones vinculadas en primer lugar con el funcionamiento general de la organización educativa, luego con resultados cualitativos de los procesos, en tercer lugar con hechos y acontecimientos que se consideran relevantes para la comunidad educativa y, por último, resultados cuantitativos de la gestión escolar, de normativa y de metas institucionales.

En este ámbito de la gestión en la organización educativa, López et al. (2003, p. 195) señalan que la autoridad y el liderazgo deben ser analizados como manifestaciones del poder, en razón a que este constituye representación específica de las relaciones sociales edificadas durante el proceso comunicativo, que genera y conserva todo sistema social. 
Al momento de enriquecer la comunicación en los centros educativos, Aramendi (2006, p. 57) propone generar y fortalecer diversas redes y estructuras de participación; identificar los posicionamientos y conductas de los docentes; supervisar la información que transita por los canales informales y formales de la entidad organizativa; eludir ambigüedades en las informaciones divulgadas; propiciar la distensión y la interrelación entre las personas; transparentar malentendidos, códigos, conceptos y lenguajes; estimular los procesos de toma de decisiones de tipo participativo; contemplar los rasgos ideológicos de las personas; apreciar la comunicación generalmente en función de su utilidad; ejecutar la redundancia, por cuanto la repetición de un mensaje de manera diferente aporta positivamente a su comprensión, y verificar el nivel de exactitud mediante el empleo de otros canales. Con relación a los modos, en la investigación de Papic (2010), de los directivos, un porcentaje levemente inferior al total, 96,2\%, expresó atender la comunicación horizontal, con el objeto de efectuar el intercambio de datos entre los miembros de un mismo nivel jerárquico, y el total, 100\%, declaró coincidencia en utilizar la comunicación horizontal para relacionar las personas y departamentos entre sí, con el objeto de mejorar la integración de los departamentos funcionales.

Para ampliar esta perspectiva, el valor instrumental de la comunicación interna como recurso gerenciable estratégico, de acuerdo con García (1998, pp. 53-54), refiere su importancia en el ámbito de la reflexión general de la organización, ya que representa un vehículo destinado a disponer de la opinión de todos los integrantes de la entidad, en términos de optimizar la productividad y competitividad mediante la mejora de las conductas de tarea y de relación interpersonal; transmitir de mejor forma, precisar y actualizar un proyecto organizacional compartido; asignar fondo y eficacia a estilos de dirección participativos y democráticos; difundir y explicitar los objetivos estratégicos e implicar a las personas en su consecución; sensibilizar al activo humano; definir los problemas y necesidades; comprometer a todos en su solución junto con integrar equipos de trabajo; incrementar la formación destinada a forjar actitudes personales; estimar la innovación y asimilar los cambios; divulgar informaciones y resultados, disponer de las ideas y opiniones de todos los integrantes, y distribuir de modo adecuado 
el trabajo, ejecutar de mejor forma las tareas, movilizar los recursos, perfeccionar el clima laboral, elevar la moral de los empleados y generar una dinámica de grupo.

Con relación al valor instrumental de la comunicación interna como recurso gerenciable estratégico, en la investigación de Papic (2010), de acuerdo con lo declarado por los directivos, la formulación y puesta en práctica de la política de comunicación organizacional en los establecimientos educativos puede estar vinculada con la finalidad de motivar a las personas para que se concentren en lo realmente importante, $84,7 \%$, y con promover que cada miembro de la organización educativa asuma su rol en la unidad o equipo de trabajo, $77 \%$.

Los alcances señalados sobre la puesta en práctica de la comunicación interna que reporta beneficios a la organización, se reafirma con el planteamiento de Cervera (2008, p. 317) acerca de indicar a las personas qué se requiere de ellas y por qué; propiciar una comprensión total entre los trabajadores de la organización; promover una mayor productividad, porque posibilita disminuir costos y perfeccionar el proceso productivo; comprometer a los empleados con los objetivos estratégicos de la entidad organizativa; reemplazar el rumor por información transparente, y entender que la sapiencia no solo radica en los niveles directivos.

Las contribuciones de la comunicación organizacional interna a la organización educativa posibilitan establecer buenas prácticas junto con compartir la responsabilidad. Acertadamente, Andrade (2005, p. 22) visualiza la comunicación como una responsabilidad compartida, porque en la organización todos se comunican y, por tanto, el hecho de comunicarse de buena manera atañe como responsabilidad a todos. Sin embargo, observa que algunas personas detentan una responsabilidad mayor, dado el lugar que ocupan o la información que manejan; en consecuencia, el desenvolvimiento de las habilidades comunicacionales en los individuos es una prioridad en la organización, sobre todo por los cambios que están sufriendo estas entidades.

A partir de lo precedente, la comunicación, según Herranz (2007, p. 9), siguiendo la cita de Bartoli (I992, p. II5), debe estar al 
servicio de la política general y enfocada a los buenos resultados de la organización.

En particular, es esencial la participación de los miembros de la organización en la comunicación, para cuyo desarrollo, según Capriotti (1998, p. 3), se debe dar cumplimiento a tres condiciones: la primera trata la confianza que deben tener los trabajadores en los directivos; la segunda aborda que los empleados gocen de la capacidad para tomar decisiones en el campo de su responsabilidad; la tercera, la convicción de los trabajadores de que, al ser escuchados y estimados por la dirección, se creará un flujo ascendente de comunicación junto con una mayor participación. En la investigación ya citada de Papic (2010), de los directivos, una amplia mayoría, 84,6\%, declaró emplear la comunicación ascendente para obtener datos sobre las opiniones de los integrantes de la comunidad educativa en el desenvolvimiento de los procesos curriculares y pedagógicos; la gran mayoría, 92,3\%, afirmó considerar la posibilidad que brinda la comunicación ascendente a los integrantes sobre la toma de conocimiento de las actividades y de los resultados de la organización, y la gran mayoría, 92,3\%, manifestó conformidad con la utilización de la comunicación ascendente con el fin de que los miembros de niveles inferiores de la organización expresen sus problemas a los niveles superiores; en tal sentido, se manifiesta una tendencia a emplear este tipo de comunicación para recoger opiniones sobre el desenvolvimiento de los procesos que conforman la gestión escolar. En cambio, de los directivos, la amplia mayoría, 77\%, expresó adhesión a contemplar la comunicación descendente como prioritaria, debido a que los integrantes de la organización se sienten partícipes de los problemas que atañen a la entidad organizativa y se observa también una tendencia positiva a este respecto.

Los tipos de comunicación interna permiten a los miembros de la organización educativa asumir su responsabilidad en la dimensión teórica y práctica. En esta última, plantea Pulgar (I999, p. 6I), en base a la cita de Pascale Weil, la función operativa o de gestión de la información, y la función de estímulo. Además, el autor refiere a Marie-Helène Westphalen y a José Luis Piñuel para señalar 
aplicaciones de la comunicación interna, tales como la de instituir una identidad organizativa capaz de aunar al conjunto del recurso humano en un ambiente de confianza y de motivación; aportar el conocimiento de la organización; imposibilitar el bloqueo interdepartamental; publicar los resultados alcanzados por la entidad organizativa; globalizar la posibilidad de comunicación, y animar la comunicación en todos los niveles institucionales.

Respecto de la relevancia de las prácticas de la comunicación organizacional interna detallo, parafraseando a Del Pozo (I997, pp. II8-I22) cuando cita a la Association Francaise de Communication Interne, las seis funciones propias de la comunicación interna: la primera consiste en investigar, a través de generar una política de escucha del ambiente social de la organización para anticiparse a alguna disfunción o generar un clima activo mediante la manifestación de diversidad de pareceres; la segunda es la de orientar, destinada a manifestar la capacidad de escuchar y concienciar acerca de los diversos aspectos de la organización, visualizados como dispositivos de gestión organizacional al interior de la comunicación interna; la tercera función es la de informar, cuyo objetivo es definir una política de información escrita, oral y audiovisual para responder a los requerimientos de información del público interno; la cuarta consiste en animar y coordinar, en términos de configurar redes de corresponsales para informar, conocer el clima social y el entorno de trabajo; la quinta función es organizar campañas para activar a un equipo de empleados y preparar campañas o eventos de comunicación interna; la sexta es formar para beneficiar el perfeccionamiento y las potencialidades de comunicación que detentan los integrantes responsables de la comunicación interna.

El ejercicio de las citadas funciones contribuye a instaurar la participación en el seno de la cultura organizacional. En el caso de la entidad educativa, señala Bataller (2006, p. 29), es factible la concreción de una verdadera participación del profesorado, en la medida en que se comprometa de forma colectiva en la gestión democrática del centro, edificando el consenso desde el disenso, la exposición de conflictos y desenvolviendo modelos participativos 
y cooperativos. Esta participación docente, explicita este autor, debe aceptar su función dinamizadora de los demás sectores de la comunidad educativa; de este modo, se debe lograr que el derecho y el deber de participar se constituya en un modo de vida en las escuelas, transformando el devenir cotidiano escolar en organización vivenciada y sentida como algo propio, comprometiendo a toda la comunidad educativa en el diálogo, consenso y puesta en práctica de modelos educativos de una manera dinámica e interactiva. Sin embargo, sugiere tener en cuenta el requerimiento de generar espacios y tiempos en las organizaciones escolares, para tornar realidad esta cultura de la participación y la gestión democráticas.

\section{Conclusiones}

En suma, se caracteriza al nuevo milenio por la globalización, la sociedad del conocimiento, la sociedad de la información, en general, y por las reformas educativas, políticas y normativas establecidas en el sistema educativo nacional, en particular. Tgodo ello configura un contexto que exige la mejora de la gestión escolar en el establecimiento educativo, con la finalidad de alcanzar la educación con calidad, con equidad y con participación, en la cual la función de comunicación organizacional interna es sustantiva, tanto para el desarrollo como para el mantenimiento de la organización educativa.

Frente al desafío de generar e institucionalizar buenas prácticas en la gestión de la organización educativa, se debe apreciar la comunicación organizacional interna como una función estratégica, que conlleva a motivar y a asumir las responsabilidades consignadas en el desempeño de los participantes de la comunidad educativa, en pos del logro del proyecto educativo institucional en el marco de la educación con calidad, equidad y participación.

En la organización educativa, la gestión de los procesos de mejora continua requieren la aplicación de un trabajo focalizado, sistemático y coordinado, que se sustente en el diseño y aplicación de políticas y estrategias de comunicación organizacional internas, bajo el prisma del derecho a la libertad de opinión y de expresión, para potenciar la concreción del derecho a la educación con calidad. 


\section{Referencias bibliográficas}

Aguaded, J. I. (2002). Nuevos escenarios en los contextos educativos. La sociedad postmoderna, del consumo y la comunicación. Revistas de la UHU Ágora digital, (3), I-I9.

Aguaded, J. I. (2010). La educación para la comunicación. Nueva alfabetización para un mundo global. Televisión: desarrollo de la creatividad e infancia, 55-70.

Álvarez, J. (2007). Comunicación interna, la estrategia del éxito. Razón y palabra. Recuperado de http://www.razonypalabra.org.mx/anteriores/n56/ index.html

Andrade, H. (2005). Comunicación organizacional interna. Proceso, disciplina y técnica. La Coruña, España: Netbiblo.

Antúnez, S. (2000). Claves para la Organización de Centros Escolares. Barcelona, España: Horsori.

Aramendi, P. (2006). Selección de centro, comunicación y concepto de calidad. Revista de educación. Obtenido de http:/ / www.uhu.es/publicaciones/ojs/ index.php/xxi/article/view/696

Asamblea General de las Naciones Unidas. (2009). Declaración Universal de los Derechos Humanos - Ley Chile. Recuperado de https://www. leychile.cl/navegar?idnorma $=1000396$

Bataller, J. (2006). La participación del profesorado. Revista cuatrimestral del consejo escolar del Estado. Recuperado de https:/ / www.academia.edu/48556I5/ Participaci\%C3\%B3n_del_Profesorado

Blejmar, B. (2006). Gestionar es hacer que las cosas sucedan. Buenos Aires, Argentina: Ediciones Novedades Educativas.

Bolívar, A. (2002). Nuestra propuesta de educación democrática. Cuadernos de Pedagogía, 317, 53-56.

Capriotti, P. (2009). Branding Corporativo: Fundamentos para la gestión estratégica de la Identidad Corporativa. Business School Universidad Mayor.

Capriotti, P. (1998). La comunicación interna. Reporte CEDD - Capacitación y Desarrollo, (I3), 5-7.

Carnicero, P. (2005). La comunicación y la gestión de la información en la institución educativa. Madrid, España: WK Educación.

Castillo, A. (2004). Nuevas tecnologías y comunicación: análisis de la intranet como soporte de comunicación interna. En J. C. Losada (Ed.), Gestión de la comunicación en las organizaciones (pp. 27I-288). Barcelona, España: Ariel S.A. 
Cervera, Á. L. (2008). Comunicación total. Madrid, España: ESIC Editores.

Chen, Z. \& Reigeluth, C. (2010). Communication in a Leadership Team for Systemic Change in a School District. Contemporary educational technology, $l(3), 233-254$.

Costa Sánchez, C. (2010). Del Press Agent a la Comunicación Estratégica. Cómo hacer que la comunicación sirva a la estrategia de gestión. Razón y Palabra. Recuperado de http://www.razonypalabra.org.mx/Costa_revisado.pdf

Delors, J. (1997). La educación encierra un tesoro. París, Francia: UNESCO.

Del Pozo Lite, M. (1997). Cultura empresarial y comunicación interna. Madrid, España: Fragua.

Del Pozo Lite, M. (2004). Identidad y cultura en la comunicación interna: herramientas estratégicas en los mercados internacionales. En J. C. Losada, Gestión de la comunicación en las organizaciones (pp. 259-270). Barcelona, España: Ariel S.A.

Emanuelli (2000). Sociedad actual e imaginarios: El marco que "influye-construye" las instituciones actuales. Revista Latina de Comunicación Social, 3 (29).

Fernández, M., Álvarez, M. y Herrero, E. (2002). La Dirección Escolar Ante Los Retos Del Siglo XXI. Madrid, España: Editorial Síntesis S.A.

Gairín, J. (1996). La organización escolar: Contexto y texto de actuación. Madrid, España: La Muralla, S.A.

Gámez, R. (2007). Comunicación y cultura organizacional en empresas chinas y japonesas. Edición electrónica gratuita. Recuperado de www.eumed.net/ libros/2007a/22I/

García Jiménez, J. (1998). La comunicación interna. Madrid, España: Ediciones Díaz de Santos, S.A.

Gento, S. y Montes, M. (2010). Cómo Elaborar un Diagnóstico de la Calidad de un Centro Educativo. Asociación Europea Liderazgo y Calidad de la educación. Recuperado de http://www.leadquaed.com/docs/artic\%20esp/ Modelo\%20de...pdf

Herranz, J. M. (2007). La comunicación dentro de las organizaciones no lucrativas. La construcción de la confianza con los ciudadanos. Razón y palabra. Recuperado de http:/ / www.razonypalabra.org.mx/anteriores/ n58/jherranz.html

Hopenhayn, M. (2006). La educación en la actual inflexión epocal: una perspectiva latinoamericana. Recuperado de http://ww2.educarchile.cl/UserFiles/ p000I/file/cr_articulos/investigador/articles-96944_hopenheimer. pdf 
López, J., Sánchez, M., Murillo, P., Lavié, J. M. \& Altopiedi, M. (2008). Dirección de centros educativos. Madrid, España: Editorial Síntesis.

Mattelart, A. (1995). La invención de la comunicación. México, D. F.: Siglo Veintiuno.

Mattelart, A. (2003). La Sociedad de la Información: El enfrentamiento entre proyectos de sociedad. Diálogos de la Sociedad d la Información, 67, 19-28.

Ministerio de Educación de Chile. (2015). Mapa de la reforma educacional. Recuperado de http://reformaeducacional.mineduc.cl/wp-content/ uploads/mapa reforma.pdf

Ministerio de Educación de Chile. (2014). Nuevos espacios educativos 2010 2013. Santiago, Chile: Ministerio de Educación.

Ministerio de Educación de Chile. (2009). Objetivos Fundamentales y Contenidos Mínimos Obligatorios de la Educación Básica y Media. Santiago de Chile: Gobierno de Chile.

Ministerio de Educación de Chile. (2005). Sistema de Aseguramiento de la Calidad de la gestión Escolar. Calidad en todas las Escuelas y Liceos. Santiago de Chile: MINEDUC.

Ordeix, E. (2009). Las relaciones públicas en la gestión de la comunicación interna. Alicante, España: Asociación de Investigadores en Relaciones Públicas.

Papic, K. (2016). La comunicación organizacional en entidades educativas. (Tesis doctoral). Málaga, España: Universidad de Málaga, Departamento de Comunicación Audiovisual y Publicidad.

Papic, K. (2010). La detección del diseño y aplicación de la planificación estratégica de la comunicación organizacional en la gestión escolar de los establecimientos educativos asimilados a la FIDE de la Sexta Región del Libertador General Bernardo O'Higgins y en condición de activos durante el año 2009. (Tesina). Málaga, España: Universidad de Málaga, Departamento de Comunicación Audiovisual y Publicidad.

Pulgar, L. (1999). Comunicación de empresa en entornos turbulentos: gestión de riesgos, reducción de las incertidumbres y supervivencia. España: Escuela Superior de Gestión Comercial y Marketing, ESIC.

UNESCO. (2010-20II). Datos mundiales de educación. Chile. Recuperado de http://www.ibe.unesco.org/fileadmin/user upload/Publications/ WDE/2010/pdf-versions/Chile.pdf

UNESCO \& UNICEF. (20I4). Mercado o garantía de derechos. Modelos en debate para la educación escolar en Chile. Santiago, Chile: UNESCO.

UNESCO. (2007). De la administración escolar tradicional a la gestión educativa estratégica.

UNESCO. Recuperado de http://ww2.educarchile.cl/UserFiles/ P000I/File/De\%20la\%20Administraci\%C3\%B3nEscolar.pdf 
Villafañe, J. (2002). Imagen positiva. Gestión estratégica de la imagen de las empresas. Madrid, España: Pirámide.

Weil, P. (1992). La comunicación global. Comunicación institucional y de gestión. Barcelona, España: Paidós Ibérica.

Zaragoza, T. (2009). Las relaciones públicas en la gestión de la comunicación interna. Sevilla, España: Asociación de Investigadores en Relaciones Públicas. 